\title{
Assessment of dispersion map impact on the digital back propagation of long-haul fibre-optic links
}

\author{
L. M. Pessoa - H. M. Salgado • I. \\ Darwazeh
}

Received: date / Accepted: date

\begin{abstract}
The back propagation algorithm is one of the best methods known for simultaneous linear and nonlinear impairment mitigation in long-haul fibreoptic links. Better understanding the full potential of such algorithm is key to improve the capacity of future links, whose design is likely to depend on the algorithm performance under different link configurations. In this paper, we carry out a novel and pertinent comparison in terms of the computational complexity requirements of both symmetric and asymmetric back-propagation implementation approaches for different dispersion map configurations, using simple single channel transmission, which results in the proposal of several design rules for the optimized deployment of ultra-long haul optical transport systems. In particular, it is concluded that dispersion unmanaged transmission is preferable in the sense of compatibility with different link design configurations as well as computational complexity requirements and maximum performance that can be achieved.
\end{abstract}

Keywords Dispersion Map · Back-propagation · Computational complexity

\section{Introduction}

Recently, the main challenge faced by optical engineers has been increasing the throughput and distance limitation of existent long-haul optical fibre transmission systems, without doing signal regeneration, as pointed by Ip et al (2008). Coherent optical systems are seen today as a key in order to accomplish these requirements because of the ability of linearly transferring the signal from the optical to the electrical domain, combined with the availability of high speed digital signal processing, which allows for the signal to be digitized and processed

L. M. Pessoa · H. M. Salgado

INESC TEC (formerly INESC Porto) and Faculty of Engineering, University of Porto, 4200-465 - Porto, Portugal

E-mail: luis.m.pessoa@inescporto.pt, hsalgado@inescporto.pt

I. Darwazeh

Department of Electronic and Electrical Engineering, University College London

Torrington Place - London WC1E 7JE, U.K.

E-mail: i.darwazeh@ee.ucl.ac.uk 
in the electrical domain. The lower price of electrical components, partly relaxed receiver requirements at high data rates and the capability of pushing the spectral efficiency limits beyond, while maximizing the power efficiency, also intensified the interest in this topic. In fact, the fibre nonlinearity plays a key role in the context of maximizing the system capacity; whereas in the linear transmission regime, the total system capacity increases with launched power, in the nonlinear regime the capacity is reduced, because fibre nonlinearity scales with the square of the power while SNR grows linearly with the power. As emphasized by Ip and Kahn (2010), the highest capacity is observed in the transition from the linear to the nonlinear regime. The compensation of both linear and nonlinear fibre impairments allows for the improvement of the highest capacity point due to an increase of the optimum launched power. As asserted by Ip and Kahn (2010), the best method known for fibre nonlinearity mitigation is backward propagation (BP), which consists in passing the received signal through a virtual fibre with opposite signs of dispersion and nonlinearity, yielding an estimate of the transmitted signal. In the absence of noise and limited computation power and provided the characteristics of the transmission channel are known, this technique can recover exactly the signal that was transmitted. It has been proposed independently by Li et al (2008) and Ip and Kahn (2008). While BP was firstly used in pre-compensation schemes, as seen in Essiambre et al (2006); Roberts et al (2006), the availability of the full electric field in a coherent optical receiver allows for BP to be used as a post-compensation technique, which is the focus of the present work. Since the BP algorithm works with the electric field of the signal, it can be applied to single carrier, wavelength division multiplexing (WDM) and orthogonal frequency division multiplexing (OFDM) signals.

Although previous works have addressed the back-propagation algorithm at the level of implementation configuration, while focusing especially on wavelength and polarization multiplexed transmission issues, such as in Goldfarb and Li (2009); Ip and Kahn (2010); Ip and Kahn (2008); Li et al (2008), no detailed analysis on the computational complexity of the BP algorithm has been performed before, comparing the symmetric and asymmetric approaches for different dispersion maps. In this work and for the first time, the computational complexity requirements of both symmetric and asymmetric BP implementation approaches are compared for different dispersion map configurations, using simple single channel transmission, resulting in the proposal of new design rules that will need to be followed to provide an optimized deployment of future optical transport systems. As history has shown, the considered optimum fiber type and link configuration of optical transport systems has been changed several times in recent years due to higher induced nonlinearities arising from the always increasing transmission rates. In this way, the present analysis is of utter importance in the sense of paving the way to achieve the highest possible capacity as well as the lowest possible energy consumption in future ultra-long haul optical transport systems, based on receiver-side BP. The remainder of this paper is organized as follows: in section 2 we introduce the numerical methods used for the fibre propagation model as well as description of the considered scenario and relevant simulation parameters. In section 3 we analyze the impact of the dispersion map characteristics in the performance of the BP algorithm. In section 4 we compare the computational complexity of both symmetric and asymmetric BP approaches, for different oversampling rates. Finally, conclusions are given in section 5 . 


\section{Fibre propagation modeling}

The propagation of a pulse $E(z, t)$ through a single mode optical fibre is governed by the nonlinear Schrödinger equation (NLSE) Agrawal (2001), which can be written in the following simplified manner:

$$
\frac{\partial E}{\partial z}=(\hat{D}+\hat{N}) E
$$

where $\hat{D}$ is a differential operator accounting for dispersion and attenuation, and $\hat{N}$ is the nonlinear operator governing nonlinear effects in the propagating signal. $\hat{D}$ and $\hat{N}$ are respectively given by:

$$
\begin{gathered}
\hat{D}=-\frac{1}{2} j \beta_{2} \frac{\partial^{2}}{\partial t^{2}}-\frac{\alpha}{2} \\
\hat{N}=j \gamma|E|^{2}
\end{gathered}
$$

where $\beta_{2}$ represents the group velocity dispersion parameter, $\alpha$ represents fibre attenuation and $\gamma$ the nonlinearity parameter. As the signal travels along the fibre, the effects of dispersion and nonlinearity interact with each other. Due to the complex interplay between these effects the NLSE has only analytic solution for some specific cases, such as solitons, being typically solved numerically by the split-step Fourier (SSF) method, introduced by Tappert in 1973 Hasegawa and Tappert (1973). However, the numerical solution given by the SSF method involves dividing the optical fibre into small segments of length $h$, wherein the effects of dispersion and nonlinearity are considered independently, which is an approximation. An exact solution to the previous equation (1) can be shown to be, after one simulation step $h$ :

$$
E(z+h, t)=\exp [h(\hat{D}+\hat{N})] E(z, t)
$$

In this work we look at two strategies of propagating the optical field through the split-step method, either by dividing the propagation from $z$ to $z+h$ into two or three steps, corresponding to the asymmetric Hayee (2008); Ip and Kahn (2008) or the symmetric Agrawal (2001) cases, respectively. While the asymmetric method induces an error proportional to $h^{2}$, the symmetric method involves an error term proportional to $h^{3}$, being therefore more accurate. In this paper we compare both methods in terms of performance and complexity in the context of back-propagation.

\subsection{Specification of simulation environment}

In this paper we consider an optical transmission channel representative of a periodically amplified long haul system. Furthermore, we consider the more accurate symmetric method for simulating the forward propagation of the signal through the optical channel, with a very small step size $h$ in order to minimize the error, by satisfying the step size requirements proposed in Hayee (2008). Fig. 1(a) shows how the long haul optical channel is implemented, having each span composed by a branch of single mode fibre (SMF), characterized by $\hat{D}_{\mathrm{smf}}$ and $\gamma_{\mathrm{smf}}$, which is followed by the optical amplifier 1 (with gain $G_{1}$ ) and then a branch of dispersion 


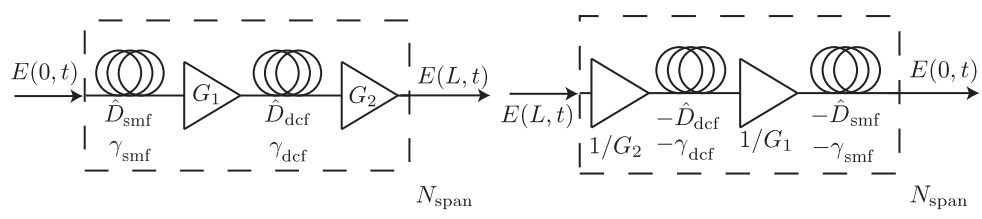

$\begin{array}{ll}\text { (a) Forward propagation } & \text { (b) Back-propagation }\end{array}$

Fig. 1 Forward and backward transmission diagrams

\begin{tabular}{|c||c||c|} 
Parameter & $\mathrm{SMF}$ & $\mathrm{DCF}$ \\
\hline Attenuation $(\alpha)$ & $0.2 \mathrm{~dB} / \mathrm{km}$ & $0.6 \mathrm{~dB} / \mathrm{km}$ \\
Dispersion $(D)$ & $17 \mathrm{ps} /(\mathrm{nm}-\mathrm{km})$ & $-80 \mathrm{ps} /(\mathrm{nm}-\mathrm{km})$ \\
Nonlinear parameter $(\gamma)$ & $0.0013 \mathrm{~m}^{-1} \mathrm{~W}^{-1}$ & $0.0053 \mathrm{~m}^{-1} \mathrm{~W}^{-1}$
\end{tabular}

Table 1 Specification of parameters for both SMF and DCF based on Ip and Kahn (2008).

compensating fibre (DCF), characterized by $\hat{D}_{\text {dcf }}$ and $\gamma_{\text {def }}$, followed by an optical amplifier 2 (with gain $G_{2}$ ). The total gain provided by the two amplifiers should be equal to the total attenuation induced by both SMF and DCF. We define the parameter $g$ as a design parameter which represents the amplification distribution factor between amplifier 1 and amplifier 2; it can be adjusted between 0 and 1 , where $g=0$ represents the total gain being lumped to amplifier 2 and $g=1$ represents the total gain being lumped to amplifier 1 . This parameter $g$ is nominally 0.5 in the simulation results presented in the following sections, which gives $G_{1}=G_{2}$. For the work presented here, the considered optical fibre parameters are summarized in Table 1 . Throughout this paper we consider a $50 \%$ RZ-QPSK modulation format, antialiasing filter set to a lowpass 5th order Butterworth with a bandwidth of $40 \%$ of the oversampling rate, a data-rate of 10 GSymbols/s, a launched power of $+4 \mathrm{dBm}$, and an optical fibre channel composed by 25 spans of $80 \mathrm{~km}$ SMF each, unless where specified otherwise. Additionally, the digitization of signals after photo-detection is considered to be performed at an oversampling rate (OR), the number of samples per symbol, at least equal to the Nyquist rate. In order to better introduce the concepts related with dispersion mapping, Fig. 2 illustrates a typical dispersion map, where typically by placing the DCFs along the link, according to a specified target residual dispersion per span (RDPS), the accumulated dispersion can be controlled. DCF is typically inserted between two optical amplifiers and can either work in a pre-compensation scheme, when it is located before the transmission fibre, and/or in a post-compensation scheme when it is located after the transmission fibre. The influence on performance induced by different dispersion map parameters will be evaluated in this work, focusing especially in the RDPS and pre-compensation.

$\mathrm{BP}$ calculates a solution for the NLSE, by passing the received signal through a virtual fibre with opposite signs of dispersion and nonlinearity, yielding an estimate of the transmitted signal, as shown in Fig. 1(b). Additionally, as back-propagation focus on the numerical solving of the NLSE, the previously introduced asymmetric and symmetric algorithms are valid.

In this work the metric used to evaluate system performance is the Q-factor, which corresponds to the electrical signal to noise ratio at the input of the receiver's decision circuit. Although the bit error rate represents the ultimate estimate of 


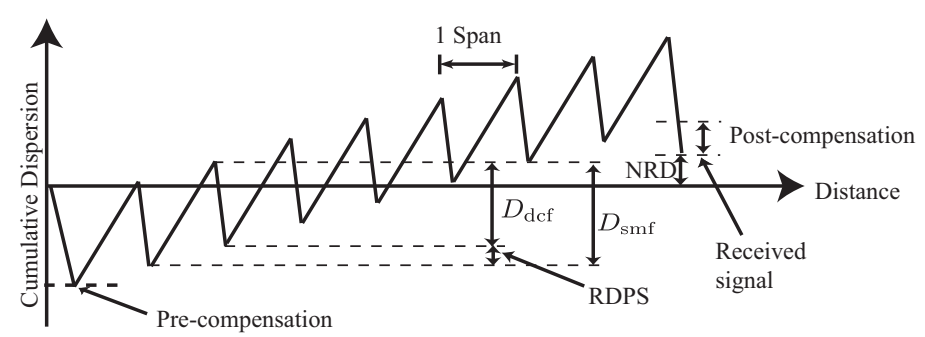

Fig. 2 Cumulative dispersion map. $D_{\text {smf }}$ and $D_{\text {dcf }}$ represent the dispersion parameters of both SMF and DCF, respectively. NRD represents the net residual dispersion.

performance, time consuming Monte Carlo simulations are required in order to capture the low probability events, and therefore the $\mathrm{Q}$ factor metric is much more adequate. In this work, the definition proposed in Lowery et al (2007) is used, where the $\mathrm{Q}$ factor is defined for QAM signaling, by extracting its value from the constellation points, using the Cartesian axes as decision thresholds.

\section{Impact of the dispersion map on back-propagation}

In fact, the main drawback with back-propagation compared to forward propagation is that the output signal can not be calculated to arbitrary precision due to the effect of noise. Therefore, the equation governing the calculation of the backpropagated electric field after one step size needs to suffer an adjustment in the nonlinear operator:

$$
E(z, t)=\exp [-h(\hat{D}+\zeta \hat{N})] E(z+h, t)
$$

The parameter $\zeta$ is the amount of nonlinearity compensated, reflecting the uncertainty of the signal amplitude used to undo the nonlinear phase rotations. For a typical transmission case, the weight of the dispersion operator is much higher than the weight of the nonlinear operator. In fact, the nonlinear operator contributes with a small perturbation on what is mainly a dispersive scenario. Therefore, if the estimation of the electric field after each BP step is accurate enough, one can perform the nonlinear phase de-rotation admitting the nonlinear operator is a good estimate of the true value, which is equivalent to set $\zeta$ near 1 , because it is likely that this phase de-rotation will make the output signal closer to the original signal. On the other hand, if the calculation of electric field is inaccurate due to noise or numerical error, it is desirable to take only a small contribution of the nonlinear operator weight for the phase de-rotation. Otherwise, an error in amplitude would be converted into a random phase rotation, leading to a even worse estimation of the signal electric field. This parameter depends on the launched power, dispersion map, oversampling rate and number of steps used for BP. The best value of $\zeta$ is found by numerical simulation, and generally is small (tends to zero) for low residual dispersion per span, and approaches 1 for dispersion unmanaged transmission (100\% RDPS). Additionally, the parameter tends to 1 for an increasing number of BP steps. 
The numerical error mentioned in the previous paragraph leads to a divergence between the output of the digital SSFM and the true solution of the propagation equation. The two main reasons leading to this phenomenon are an insufficiently small step size length, and the sample rate not satisfying Nyquist's criterion, when taking into account the new frequencies generated by the nonlinearity. Ip and Kahn (2008) has found that an oversampling rate of three may be required for backpropagation in order to avoid aliasing effects, due to the electric field being third order on the propagation equation. Furthermore, Goldfarb and Li (2009) showed that any inaccuracies in the representation of the FIR linear filter responsible for dispersion compensation operation, would result in a numerical error, due to error accumulation at each iteration.

\subsection{Performance impact induced by $\zeta$ parameter}

At this point, it is relevant to gain insight on the influence of the parameter $\zeta$ on system performance, while varying the value of RDPS. Furthermore, since the DCF also contributes to both dispersion and nonlinearity phenomena, it is also useful to note the system sensitivity to these parameters, specifically, how performance is affected by turning off these parameters in the simulations. Fig. 3 demonstrates three sets of results for $\zeta \in\{0,0.5,1\}$, for a typical scenario referred as "A" (solid lines), for a scenario "B" where the DCF dispersion effect is turned off (dashed lines), while maintaining its nonlinearity and finally for the case "C" where the DCF nonlinearity effect is turned off (dotted lines), while maintaining its dispersion. note that for scenario "B" the effective residual dispersion per span does not correspond to its nominal value, since the dispersion effect is not cancelled by the DCF. However, the objective is to investigate the interplay between dispersion and nonlinearity, in order to quantify the contribution of each effect separately. These simulations were performed using an oversampling rate of $O R=3$ and 3 sections per span.

In the first place, the results with $\zeta=0$ should be looked at. If starting from the $100 \%$ RDPS point, considering scenario "A", one can see that the performance degrades as the RDPS decreases, since the DCF length is increased, which on its turn increases the loss and DCF nonlinearity, thus leading to a worse performance. Additionally, a reduction of SMF dispersion (by adding DCF) can increase the impact of nonlinear effects. In fact, the presence of dispersion reduces the impact of nonlinearity because it induces the noise to walk-off from the signal, reducing the nonlinear interactions between them Ip and Kahn (2008). However, in spite of these mentioned effects that lead to performance worsening, one might note that when approaching $20 \%$ RDPS the Q factor starts improving continuously up to $0 \%$ RDPS. This indicates that another effect starts dominating, which is the fact that the nonlinear phase rotations occur closer to points of zero dispersion, therefore affecting the signal at instants where its original constellation form is nearly recovered. Therefore, at the receiver, if no nonlinear compensation is performed along with dispersion reversal (for $\zeta=0$ ), the signal constellation is still reasonably acceptable, exhibiting a spiral effect from nonlinear phase rotations, as shown in inset constellations of Fig. 3. On the other hand, when nonlinear phase rotations systematically occur at points of high dispersion (for higher RDPS values), nonlinearity impacts a signal which is largely distorted by dispersion, therefore, 


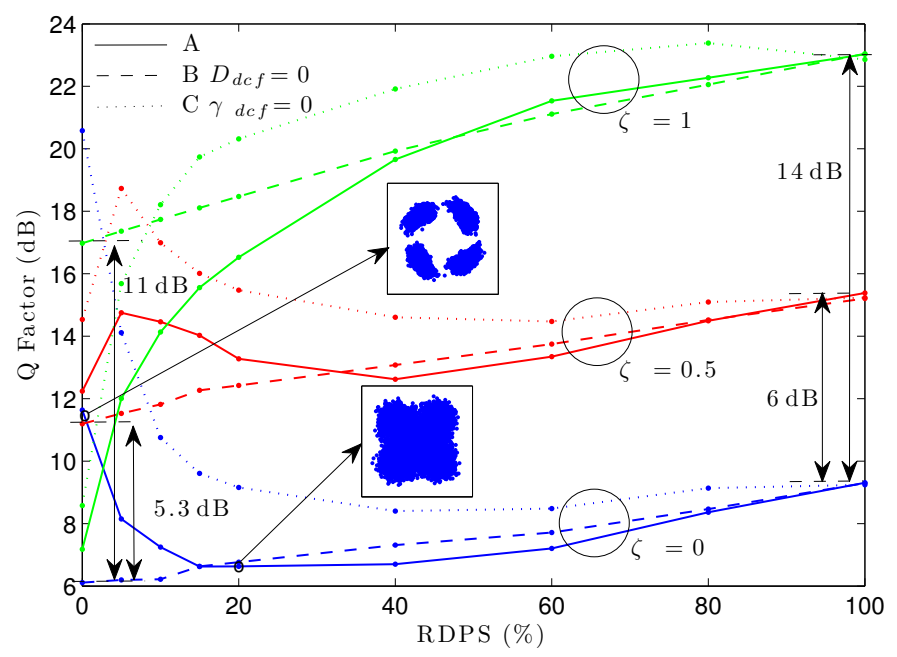

Fig. 3 Performance comparison on the influence of $D_{\mathrm{dcf}}=0$ (dashed lines) and $\gamma_{\mathrm{dcf}}=0$ (dotted lines)

after dispersion reversal the signal remains still very distorted, as also shown by the inset constellations of Fig. 3.

Scenario "B" gives slightly better performance than "A" for high RDPS values. While in "A", the increase of DCF length leads to a reduction of the SMF dispersion effect and consequent reduction of the advantageous noise walk-off effect, in "B" there is no reduction of the SMF dispersion because DCF dispersion is not activated, and therefore this effect does not occur again. Additionally, in "B", there is no performance improvement near $0 \%$ of RDPS, because the effect of SMF dispersion cancellation does not happen. In this way, the performance linearly degrades from $100 \%$ to $0 \%$ RDPS, with the only effect contributing to this degradation being the DCF loss (requiring an increased level of amplification, and therefore higher noise) and also the induced nonlinearity. Finally, for scenario "C", the best performance compared to scenarios "A" and " $\mathrm{B}$ " is obtained. When comparing to scenario "A", the tendencies are the same (performance degradation for high RDPS values and performance improvement for low RDPS values), but a general improvement is obtained which goes from $0 \mathrm{~dB}$ at $100 \%$ to approximately $8 \mathrm{~dB}$ at $0 \%$ RDPS, due to the impact of the DCF nonlinearity being removed. The decreasing performance when RDPS goes from $100 \%$ downwards is due to the above mentioned reduction of the noise walk-off effect.

Regarding the results for $\zeta=0.5$, the curves tendencies are similar to the $\zeta=0$ case, the $\mathrm{Q}$ factor being shifted by $6 \mathrm{~dB}$ at $100 \%$ RDPS, due to the impact of the back-propagation algorithm on the compensation of the nonlinear phase. However, when approaching $0 \%$ RDPS, the performance does not keep the $6 \mathrm{~dB}$ of advantage, for all scenarios. In scenario " $\mathrm{B}$ " it is approximately $5.3 \mathrm{~dB}$ above at RDPS $=0 \%$, showing that the penalty of the BP algorithm in compensating the DCF nonlinearity is approximately $0.7 \mathrm{~dB}$. However, for scenarios "A" and "C" where the DCF dispersion is activated, the $\mathrm{BP}$ algorithm does not bring significant 
advantages, being even worse for case "C". This is due to the phase matching phenomenon, which consists in phase de-rotations adding coherently after each span (near zero RDPS), and proportionally to power, leading to a degradation of the $\mathrm{Q}$ factor. Therefore, as the RDPS deviates from $0 \%$ the phase additions loose coherence, due to the averaging effects of chromatic dispersion, leading to a performance improvement.

Finally for $\zeta=1$, as expected from the previous explanation, the performance is the best at $100 \%$ RDPS, compared to the results for $\zeta=0$ and $\zeta=0.5$. For $0 \%$ RDPS, the performance is only improved for scenario "B". In this scenario, the performance gain is approximately $14 \mathrm{~dB}$ at $100 \%$ RDPS and $11 \mathrm{~dB}$ at $0 \%$ RDPS, compared to the $\zeta=0$ case.

The above presented results suggest that the optimum value of $\zeta$ reduces with RDPS reduction, which indicates that the compensation uncertainty is higher for lower RDPS values, where the low values of dispersion lead to an higher impact of noise and phase matching.

\subsection{Analysis of pre-compensation impact}

The pre-compensation reflects the amount of dispersion placed at the transmitter in order to shift the dispersion map in the positive or negative direction depending on positive or negative dispersion being used for that purpose, respectively, which can be visualized by recalling Fig. 2, where a negative amount of dispersion is used for pre-compensation. Since chromatic dispersion and nonlinearity interact with each other, the amount of pre-compensation is expected to have an impact on the resulting performance. In Fig. 6 the $\mathrm{Q}$ factor performance for the specific pre-compensation values of $P_{c}=\{0,-1 / 2,-1,-2\}$ relative to the dispersion of a single span of SMF, is shown for the cases of $\zeta=0$ (a) where the nonlinearity is not compensated, and $\zeta=$ optimum (c), where nonlinearity is compensated using the BP algorithm with a tuned value of $\zeta$. In (b) and (d), the analysis is analogous to (a) and (c), respectively, but the DCF nonlinearity is turned off in order to facilitate the analysis of the results. The considered number of sections per span for the BP algorithm was set to 5 .

Regarding the non-compensated cases, it can be concluded that using a more negative value of pre-compensation (to a certain extent) results in a better $\mathrm{Q}$ factor, especially for lower RDPS values, where the cumulative dispersion has a small variation from span to span, therefore crossing the zero dispersion point more times. It should be noted that as the pre-compensation value decreases, the Q factor curves start exhibiting a maximum, which is related with the RDPS value for which the average cumulative dispersion is closer to zero. In case (b) where $\gamma_{\mathrm{dcf}}=0$ the results are generally better as expected, due to the absence of DCF nonlinearity. Additionally, the fact that the best $\mathrm{Q}$ factor at zero RDPS is for zero pre-compensation indicates that it is at the beginning of each span that nonlinearity has stronger impact due to the higher signal power after amplification, as expected. Therefore, at zero RDPS, when moving from zero pre-compensation to $P_{c}=-1 / 2$, the point where SMF nonlinearity is stronger moves away from zero dispersion, leading to a worse performance.

Considering the cases of compensated nonlinearity (c) and (d), one can observe that the $\mathrm{Q}$ factor is essentially affected by the amount of pre-compensation 


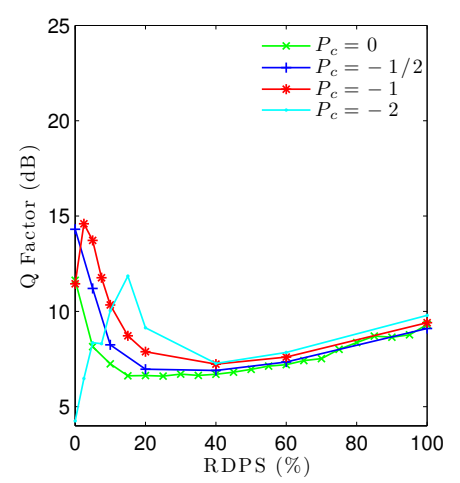

(a) $\zeta=0$

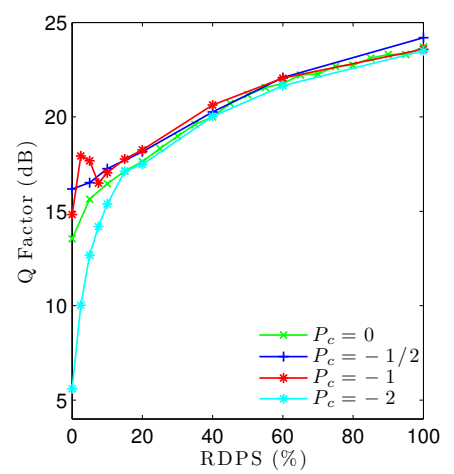

(c) $\zeta=$ optimum

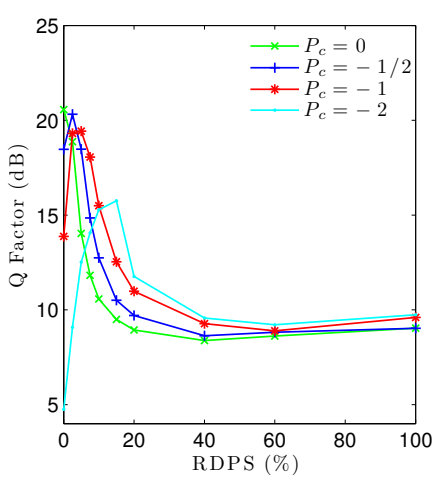

(b) $\zeta=0$ and $\gamma_{\mathrm{dcf}}=0$

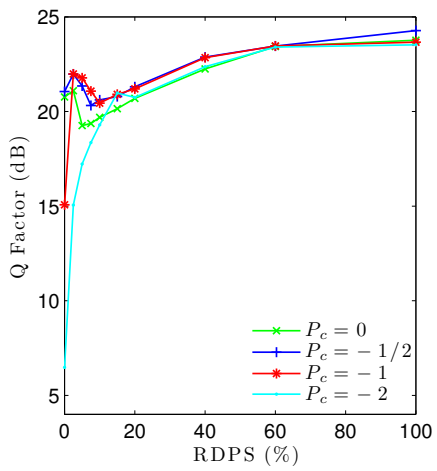

(d) $\zeta=$ optimum and $\gamma_{\mathrm{dcf}}=0$

Fig. 4 Q Factor versus RDPS for varying amounts of pre-compensation, specifically $P_{c}=$ $\{0,-1 / 2,-1,-2\}$ relative to the dispersion of a single span of SMF, with (a) $\zeta=0$, (b) $\zeta=0$ and $\gamma_{\mathrm{dcf}}=0,(\mathrm{c}) \zeta=$ optimum and (d) $\zeta=$ optimum and $\gamma_{\mathrm{dcf}}=0$

for low RDPS values. There is a small performance improvement in $\mathrm{Q}$ factor with reducing RDPS (below $10 \%$ ), for $P_{c}=-1$ in (c) and $P_{c}=\{-1 / 2,-1\}$ in (d), which occurs due to the cumulative dispersion map being near zero at those specific pre-compensation values. As seen previously, this leads to a high $\mathrm{Q}$ factor without compensation, and subsequently the observed improvement in the case of compensated nonlinearity is relative to this non-compensated case. In fact, the performance difference from the compensated to the non-compensated case does not vary significantly with pre-compensation. Therefore the maximum achievable Q factor for low RDPS values is possible with a pre-compensation between $P_{c}=0$ and $P_{c}=-1$.

\subsection{Performance impact induced by parameter $g$}

So far the parameter $g$ has been considered as 0.5 for simplicity. However, it is of relevance to analyze the impact of varying the parameter $g$ for the long haul amplified link in consideration. Fig. 5 presents the results on the performance impact of $g$ 


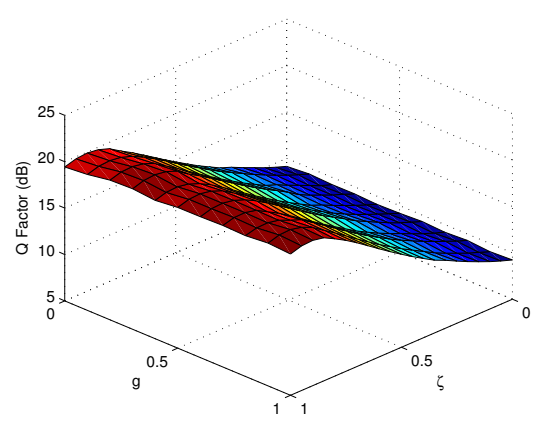

(a) $100 \%$ RDPS, $\mathrm{LP}=+4 \mathrm{dBm}$

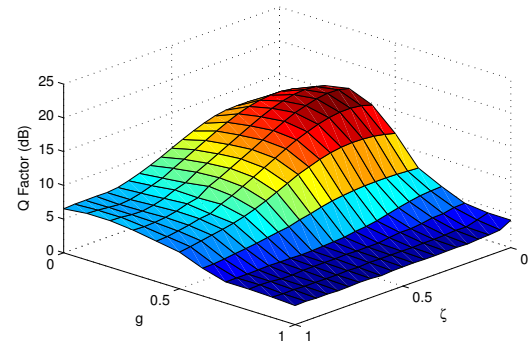

(c) $0 \%$ RDPS, LP $=+4 \mathrm{dBm}$

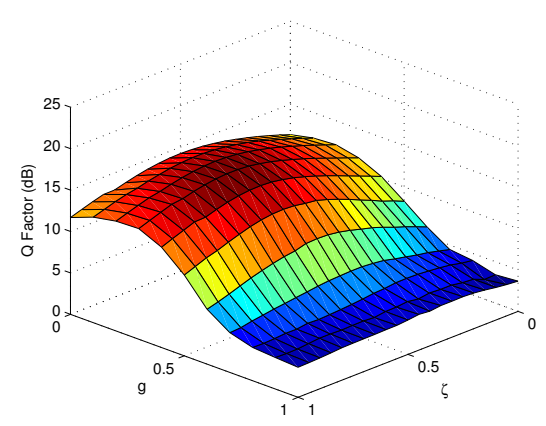

(b) $5 \%$ RDPS, LP $=+4 \mathrm{dBm}$

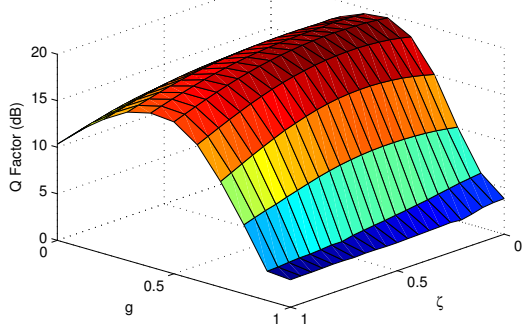

(d) $0 \%$ RDPS, LP $=-2 \mathrm{dBm}$

Fig. 5 Q factor versus $g$ factor versus $\zeta$ parameter at 10 GSymbols/s for (a) $100 \%$, (b) $5 \%$ and (c) $0 \%$ RDPS with a launched power of $+4 \mathrm{dBm}$ and (d) $0 \%$ RDPS with a launched power of $-2 \mathrm{dBm}$

as a function of $\zeta$, for $\operatorname{RDPS}=\{0,5,100\} \%$ at 10 GSymbols/s. Although in an ideal optical transmission the optimum $g$ value tends towards the unity regardless of the RDPS value, after including linear and nonlinear impairments with subsequent BP this holds only at $100 \%$ RDPS. In fact, for $100 \%$ RDPS the performance does not depend on the parameter $g$, since, for this specific case, the DCF is not present. However, for lower values of RDPS the optimum $g$ decreases to approximately 0.3 ; although not shown here, a similar result was observed for 25 Gsymbols/s. This is due the to fact that the DCF nonlinearity becomes significant for lower values of RDPS and therefore it is preferable to provide higher amplification to the signal after the DCF rather than before, in order to avoid its nonlinearities. This effect is also proportionally dependent on the signal launched power. Considering the worst RDPS scenario of $0 \%$ (when DCF has the most significant impact), we evaluated the resulting performance as a function of $g$ and $\zeta$ for different launched powers. For low launched powers $(-8 \mathrm{dBm})$ the optimum $g$ factor is approximately 0.7 , indicating that a higher gain for amplifier 1 is beneficial, since the DCF nonlinearity is not significant at this launched power, leading to an increase on the OSNR, before the DCF induces attenuation on the signal. Furthermore, as the launched power increases, the system performance increasingly moves towards the nonlinear regime, and therefore the optimum $g$ moves towards 0 . The optimum values found 


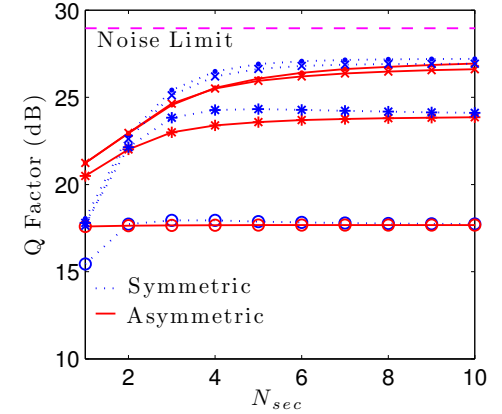

(a) $\mathrm{RDPS}=100 \%$

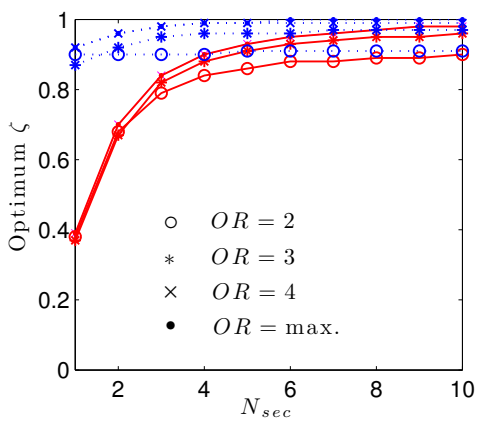

(c) $\mathrm{RDPS}=100 \%$

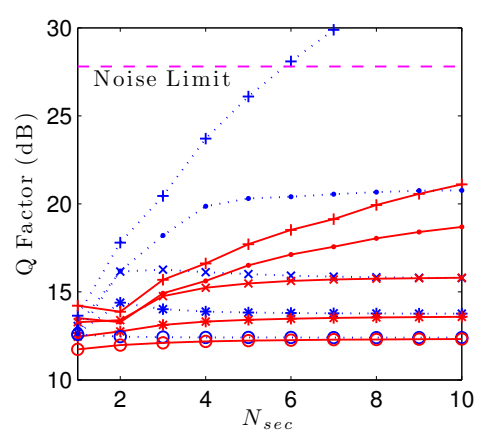

(b) $\mathrm{RDPS}=0 \%$

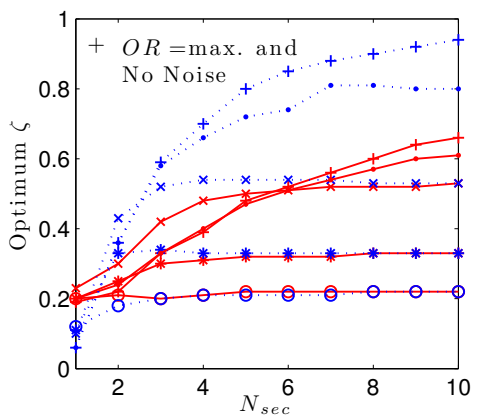

(d) $\mathrm{RDPS}=0 \%$

Fig. 6 Comparison on Q Factor and optimum $\zeta$ parameter versus the number of sections of the BP algorithm at 10 GSymbols/s at both $100 \%$, (a) and (c) as well as $0 \%$ RDPS, (b) and (d).

were $g=0.5$ for $-2 \mathrm{dBm}$ (plotted in Fig. $5(\mathrm{~d})$ ), $g=0.3$ for $+4 \mathrm{dBm}$, and $g=0.2$ for $+6 \mathrm{dBm}$, which confirms the increasing impact of DCF nonlinearity.

\section{Analysis of BP step size requirements}

Ip and Kahn Ip and Kahn (2010); Ip and Kahn (2008) have discussed that the step size requirements for $\mathrm{BP}$ are much more relaxed than those required to model communication systems (forward propagation). They have shown that the step size can take values in such a range that the numerical error stays small compared to the impact of AWGN. The step size is the most significant parameter in determining the complexity of the BP algorithm. Therefore, an extensive analysis of the effect of varying the number of sections used in the back-propagation algorithm was performed, for the limit cases of $100 \%$ and $0 \%$ RDPS as shown in Fig. 6.

The careful analysis of these plots carries a lot of useful information, that allows one to understand the influence of the oversampling rate, RDPS value and type of algorithm used, while gaining sensitivity to the importance of each of these parameters. The maximum number of sections used was 10, because it is possible to achieve a stable Q factor within this limit, regardless of the oversampling rate. First of all, it is clear that an oversampling rate of two is insufficient, in all cases, 
since the performance is clearly poor in terms of Q factor, and additionally the $\zeta$ parameter stays away from one indicating that numerical error has a significant impact. An oversampling rate of three is preferable, since it provides better results, but the RDPS value should be analyzed. In fact, for low values of RDPS, it is clear that an oversampling rate of four tends to be necessary. The results corresponding to using all of the samples available, provide an indication on how far is the performance of each of the different oversampling rate from the limit. In particular, for $100 \%$ RDPS case, an oversampling rate of four has practically the same performance as the limit, indicating there is no benefit in increasing the oversampling rate beyond this value. However, this does not hold for cases of low RDPS, where more than four times of oversampling would be required to achieve a performance near the limit, which would lead to high computational complexity. For the specific case of $0 \%$ RDPS, an additional result is presented, corresponding to the scenario of turning off the AWGN in the simulation, for the maximum oversampling rate. The results for this case indicate that the $\mathrm{Q}$ factor improves steadily with the increasing number of sections, the improvement being even more pronounced for the more precise symmetric algorithm. This indicates that it is effectively the presence of noise that leads to numerical error in the simulation and that its impact is higher on the symmetric algorithm performance than on the asymmetric, which can possibly be attributed to the iterative nature of the symmetric algorithm. In fact, the presence of noise (and consequent amplitude fluctuations) leads to phase de-rotation values being proportional to the noisy amplitude, which makes the algorithm solution to diverge from the original value. The noise does not have a significant impact for uncompensated transmission, because the dispersion effect leads the noise to walk-off from the signal, while for dispersion managed transmission the noise stays in band with the signal, increasing nonlinear interactions between noise and signal.

The performance analysis as a function of the number of sections used in the $\mathrm{BP}$ algorithm was also carried out for a data-rate of 25 GSymbols/s. Although not shown in the results, similar conclusions to the $10 \mathrm{GSymbols} / \mathrm{s}$ analysis could be obtained. It could be observed that a higher number of sections is required for the higher data-rate of $25 \mathrm{GSymbols} / \mathrm{s}$, compared to the $10 \mathrm{GSymbols} / \mathrm{s}$, as expected from the step size requirements equation proposed in Hayee (2008), which translates into a smaller step the higher the signal bandwidth. Furthermore, for low RDPS regimes, the performance at 25 GSymbols/s benefits from higher $\zeta$ values than for $10 \mathrm{GSymbols} / \mathrm{s}$, which can be attributed to the higher intensity of chromatic dispersion (due to the higher data-rate), which attenuates the noise impact and the phase matching issue.

\subsection{Computational complexity comparison}

The analysis provided in the previous section is useful in the sense of understanding the influence of the number of sections for both symmetric and asymmetric algorithms, for different oversampling rates. However, based on those results, one may find difficult to determine how the complexity of each algorithm compares with each other while looking at system performance. Therefore, it is of relevance to provide a means of comparing the performance gain with the complexity increase, as a function of the oversampling rate. This is provided in Fig. 7. One 


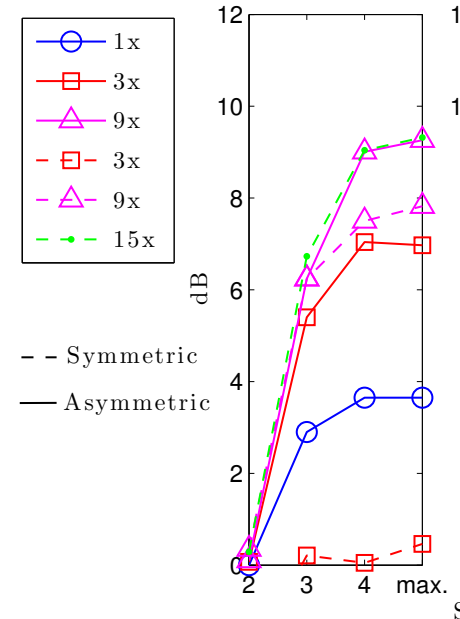

(a) $\mathrm{RDPS}=100 \%$

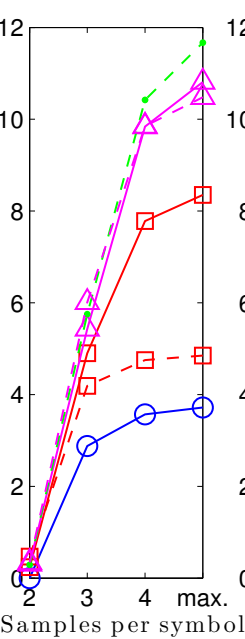

(b) $\mathrm{RDPS}=5 \%$

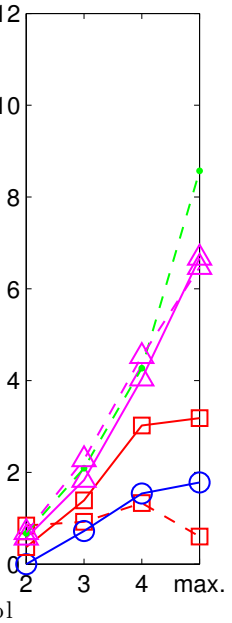

(c) $\mathrm{RDPS}=0 \%$

Fig. $7 \mathrm{Q}$ factor improvement referred to the performance of the asymmetric algorithm with 2 samples per symbol (legend "A $1 \mathrm{x}$ ") as a function of the oversampling rate at 10 GSymbols/s, comparing several computation complexity efforts of both symmetric and asymmetric algorithms at (a) 100\% RDPS, (b) 5\% RDPS and (c) $0 \%$ RDPS

important remark is that the computational complexity of the symmetric algorithm is three times higher than its asymmetric counterpart, essentially because of the iterative operation associated with the splitting of the dispersion operator in two parts, as described inAgrawal (2001). Additionally, the asymmetric algorithm with 2 samples per symbol has been used as reference, defined as having " $1 \mathrm{x}$ " of computational complexity, which corresponds to using a single section (or equivalently a span length section). Additionally, the results represented by dashed lines correspond to the symmetric algorithm, using 1,3 , and 5 sections, which translates in a " $3 \mathrm{x}$ ", " $9 \mathrm{x}$ " and " $15 \mathrm{x}$ " complexity due to the three times factor of comparison to the asymmetric case. The results show that the asymmetric algorithm outperforms the symmetric algorithm for similar complexity levels, with the performance advantage gradually vanishing as both the RDPS decreases and the complexity increases.

\section{Conclusions}

The back-propagation algorithm performance was studied in detail, and optimum design rules were determined from simulation results, where the dispersion map, oversampling rate and computational complexity were the parameters of interest. It was concluded that the optimum value of the nonlinear phase de-rotation parameter, $\zeta$, varies with the RDPS, indicating that the compensation uncertainty is higher for lower RDPS values, where the low values of dispersion lead to a higher impact of noise and phase matching issues. Moreover, the analysis of dispersion pre-compensation has shown an optimum value of dispersion between $P_{c}=0$ and $P_{c}=-1$, relative to the dispersion of a single span of SMF. Furthermore, the 
influence of the amplifier gain splitting ratio $g$ was assessed, its optimum value decreasing with increasing launched power, for low RDPS values, due to the fact that the DCF nonlinearity becomes significant and therefore it is preferable to provide higher amplification to the signal after the DCF rather than before, in order to avoid its nonlinearities.

Concerning the analysis of computational complexity, it was found that oversampling requirements increase with decreasing RDPS, which results from the noise impact on the simulation numerical error. Additionally, the asymmetric algorithm was found to out-perform the symmetric algorithm for similar complexity levels, with the performance difference gradually vanishing as the RDPS decreases. From the comparison of results at 10 GSymbols/s and 25 GSymbols/s, it was concluded that an higher number of sections is required for higher bandwidth signals, while higher optimum $\zeta$ values are obtained with the higher data-rate signals due to the stronger impact of chromatic dispersion.

As a final remark, it can be concluded that dispersion unmanaged transmission is preferable in the sense of compatibility with different link configurations (namely, different specifications of pre-compensation and $g$ parameter) as well as computational complexity requirements and maximum performance that can be achieved.

\section{References}

Agrawal G (2001) Nonlinear Fiber Optics, 3rd edn. Academic Press

Essiambre R, Winzer P, Wang X, Lee W, White C, Burrows E (2006) Electronic predistortion and fiber nonlinearity. IEEE Photonics Technology Letters 18(17):1804-1806

Goldfarb G, Li G (2009) Efficient backward-propagation using waveletbased filtering for fiber backward-propagation. Optics Express 17(11):8815-8821

Hasegawa A, Tappert F (1973) Transmission of Stationary Nonlinear Optical Physics in Dispersive Dielectric Fibers I: Anomalous Dispersion. Appl Phys Lett 23(3):142-144

Hayee Q (2008) Symmetrized split-step Fourier scheme to control global simulation accuracy in fiber-optic communication systems. J Lightwave Technol 26:302-316

Ip E, Kahn J (2010) Fiber Impairment Compensation Using Coherent Detection and Digital Signal Processing. Journal of Lightwave Technology 28(4):502-519

Ip E, Kahn JM (2008) Compensation of Dispersion and Nonlinear Impairments Using Digital Backpropagation. Journal of Lightwave Technology 26:3416-3425, DOI 10.1109/JLT.2008.927791

Ip E, Lau A, Barros D, Kahn J (2008) Coherent detection in optical fiber systems. Opt Express 16:753-791

Li X, Chen X, Goldfarb G, Mateo E, Kim I, Yaman F, Li G (2008) Electronic post-compensation of WDM transmission impairments using coherent detection and digital signal processing. Opt Express 16:880-888

Lowery A, Du L, Armstrong J (2007) Performance of optical OFDM in ultralonghaul WDM lightwave systems. Lightwave Technology, Journal of 25(1):131-138

Roberts K, Li C, Strawczynski L, O’Sullivan M, Hardcastle I (2006) Electronic precompensation of optical nonlinearity. IEEE Photonics Technology Letters 18(2):403-405 